\title{
Implementation of 2x2 MIMO-OFDM System using Universal Software Radio Peripherals
}

\author{
Dr. Yedukondalu Kamatham ${ }^{1}$ and Naveen Talati ${ }^{2}$ \\ ${ }^{1}$ Professor, CVR College of Engineering/ECE Department, Hyderabad, India \\ Email: kyedukondalu@gamil.com \\ ${ }^{2}$ PG Scholar, CVR College of Engineering/ECE Department, Hyderabad, India \\ Email: naveenthalati741@gmail.com
}

\begin{abstract}
The 2x1 and 2x2 Multiple Input Multiple Output (MIMO) systems with Orthogonal Space-Time Block Code (OSTBC) are implemented using Universal Software Radio Peripherals (USRPs) and LabVIEW. Bit Error Rate (BER) is estimated and compared for both systems using different Mary Quadrature Amplitude Modulation (QAM) i.e. 4 QAM, 16 QAM, 64 QAM. The BER is reduced for a $2 \times 2$ MIMO than a 2x1. To improve spectral efficiency, Orthogonal Frequency Division Multiplexing (OFDM) is combined with MIMO configuration, known as MIMO-OFDM. 2×2 MIMO-OFDM with OSTBC is implemented with M-ary QAM and N IFFT (32, 64, 128 IFFT). Among these, better BER performance is observed for $2 \times 2$ MIMO with 64-IFFT. The MIMO-OFDM is used in Worldwide Interoperability for Microwave Access (WiMAX) which is the standard of IEEE 802.16.
\end{abstract}

Index Terms: Multiple Input and Multiple Output (MIMO), Orthogonal Frequency Division Multiplexing (OFDM), Bit Error Rate (BER), Universal Software Radio Peripheral (USRP)

\section{INTRODUCTION}

In wireless communications systems, the current technology demands reliability and high data rates with improved spectral efficiency and Quality of Service (QoS) with various users at the same time. Improved spectral efficiency can be offered by Orthogonal Frequency Division Multiplexing (OFDM) and Multiple Input Multiple Output (MIMO) offers high data rates with spatial multiplexing and reliability with spatial diversity gain [1-2]. The reduced Bit Error Rate (BER) is obtained by diversity gain which provides several copies of transmitted signals at the receiver. Spatial multiplexing is also a MIMO technology that transmits different data streams over multiple antennas simultaneously without an increase in bandwidth results in high data rates. A decade ago, extensive research on MIMO has been started. In the $1990 \mathrm{~s}$, the Alamouti scheme was proposed in the literature [3] with simple transmit diversity scheme for two transmit antenna systems and the spatial multiplexing scheme was first proposed in literature [4]. The principle behind the spatial multiplexing scheme is as follows: the transmission of high data rate of bit sequence is de-multiplexed into a low data rate of $\mathrm{M}$ subsequences, is modulated and transmitted over different transmit antennas simultaneously using the same frequency band. The interference cancellation algorithm is used to separate transmitted sequences at the receiver [5].
The combined technology of MIMO with OFDM gained more attention due to high data rates with spectral efficiency. OFDM is a popular technology used in wireless communications systems because of multi-carrier transmission in which a wide bandwidth is split into parallel ' $n$ ' sub-bands with sub-carriers i.e. each subcarrier experiences a narrow band. The advantage of OFDM is immunity to multipath fading and Inter Symbol Interference (ISI). ISI occurs when modulated symbol time is less than the delay-spread results in a non-deductible error floor which reduces the performance of the system significantly. To overcome ISI, equalization [6] is carried out on the receiver side. Various detection algorithms are available at the receiver side for the frequency flat fading that is categorized by different trade-offs between performance and complexity. To reduce the complexity, a linear receiver is a better choice.

This paper focuses to achieve better BER performance with $2 \times 1$ and $2 \times 2$ MIMO systems. In this paper, a $2 \times 2$ MIMO-OFDM system is implemented with the help of Universal Software Radio Peripherals (USRPs) and LabVIEW. BER performance is compared by increasing the FFT size of OFDM and with different M-QAM modulations. LabVIEW is a graphical programming language that provides inbuilt blocks like Fast Fourier Transform (FFT) and Inverse Fast Fourier Transform (IFFT) which are used to sample the real-time signals [7]. National Instruments (NI) USRPs are the Software Defined Radios (SDRs), which is a range of programmable Field Programmable Gate Array (FPGA) based Tx/Rx SDR made by Ettus research. With high speed and resolution ADCs and DACs, these high-quality devices take a modular approach and allow us to use a selection of a transmitter, a receiver, and transceiver. Radio Frequency (RF) daughter boards that are optimized to work at various frequencies [8].

The rest of the paper is organized as follows: Section II introduces an implementation of a simple MIMO system. The implementation of a $2 \times 2$ MIMO-OFDM system is presented in detail in section III. Section IV discusses the results and discussions. Section V deals with the conclusion of the paper.

\section{IMPLEMENTATION OF THE MIMO SYSTEM}

MIMO communications system developed in the year 1990s and they became an integral part of the present wire 
or wireless communications systems. Multipath propagation is the advantage in MIMO, various paths are formed due to bounces of vehicles, buildings and other obstacles and reach the receiver in different directions and at different times, this increases the receiver signal capturing power [9].

The transmitted sequence bits are modulated using M-ary Quadrature Amplitude Modulation (M-QAM), two parallel streams and in that first stream transmit through $\mathrm{Tx} 1$ antenna and other is through Tx2 antenna using Alamouti Space Time Block Code encoding scheme. Orthogonal Space-Time Block Code (OSTBC) is a superior kind of Space-Time Block Code (STBC) in which each column in the matrix of this code is orthogonal to individuals. Full diversity is achieved over the coherent channel at low computational cost using STBC. The STBC encoding and decoding procedure for a $2 x 1$ MIMO is clearly shown in Fig 1 , where $\mathrm{p} 1, \mathrm{p} 2, \mathrm{p} 3, \mathrm{p} 4$ are transmitting symbols, the same pair of symbols $\mathrm{p} 1$ and $\mathrm{p} 2$ transmitted through two different antennas twice over two symbol periods [10]. The transmitted symbols at the physical antenna have a little variation at each symbol timing. At the symbol time $t 1$ direct symbols $\mathrm{p} 1$ and $\mathrm{p} 2$ are transmitted and at the symbol time $\mathrm{t} 2$ modified symbols $\mathrm{p} 2 *$ and $\mathrm{p} 1 *$ are transmitted as shown in Table I. Where $\mathrm{p} 1 *$ and $\mathrm{p} 2 *$ are conjugate of $\mathrm{p} 1$ and $\mathrm{p} 2$ respectively. $\mathrm{h} 1$ and $\mathrm{h} 2$ are channel coefficients represented with noise and fading. If there is no fading, interference, and noise present in the channel, the receiver decodes the transmitted data successfully without complicated transmission but in real-time, it is not possible. $\mathrm{y} 1$ and $\mathrm{y} 2$ are the signals at the receiver antennas 1 and 2 respectively [11]. The receiver does not process the data at each symbol. It waits till it receives two received symbols and combined them to decode and separate the symbols $\mathrm{p} 1$ and $\mathrm{p} 2$ with the help of weight vectors. The decoding procedure is as follows:

TABLE I.

SYMBOL TRANSMISSION AT THE TRANSMITTER SIDE

\begin{tabular}{|c|c|c|}
\hline Antenna's & Time slot 1 & Time slot 2 \\
\hline 1 & $p_{1}$ & $-p{ }^{*}$ \\
\hline 2 & $p_{2}$ & $p_{1}{ }^{*}$ \\
\hline
\end{tabular}

The received signal is represented as

$$
\check{y}=H \bar{p}+\bar{n}
$$

Where $\mathrm{Y}$ is the signal received at the receiver, $\mathrm{H}$ is the channel matrix, $p$ is the symbols transmitted at the transmitter and $\mathrm{n}$ is the noise.

The received signal at two-time slots is given by:

$$
\left[\begin{array}{l}
y_{1} \\
y_{2}
\end{array}\right]=\left[\begin{array}{ll}
h_{1} & h_{2}
\end{array}\right]\left[\begin{array}{cc}
p_{1} & -p_{2}{ }^{*} \\
p_{2} & p_{1}{ }^{*}
\end{array}\right]+\left[\begin{array}{l}
n_{1} \\
n_{2}
\end{array}\right]
$$

Received signal at time $\mathrm{t} 1$ is given by:

$\left[y_{1}\right]=\left[\begin{array}{ll}h_{1} & h_{2}\end{array}\right]\left[\begin{array}{l}p_{1} \\ p_{2}\end{array}\right]+\left[n_{1}\right]$

Received signal at time $\mathrm{t} 2$ is given by:

$\left[y_{2}\right]=\left[\begin{array}{ll}h_{1} & h_{2}\end{array}\right]\left[\begin{array}{c}-p_{2}^{*} \\ p_{1}^{*}\end{array}\right]+\left[n_{2}\right]$

\section{Where}

y1 and $y 2$ are the received signals at different time

\begin{abstract}
$\mathrm{P} 1 *$ is the conjugate of $\mathrm{p} 1$ symbol $\mathrm{P} 2 *$ is the conjugate of $\mathrm{p} 2$ symbol $\mathrm{n} 1$ and $\mathrm{n} 2$ are noise
\end{abstract}

Simplifying the equation (4) to obtain $y_{2}{ }^{*}$

$$
y_{2}{ }^{*}=\left[h_{2}{ }^{*}-h_{1}{ }^{*}\right]\left[\begin{array}{l}
p_{1} \\
p_{2}
\end{array}\right]+n_{2}{ }^{*}
$$

Combining the equations (3) and (5) to get the orthogonal channel matrix as:

$\left[\begin{array}{c}y_{1} \\ y_{2}{ }^{*}\end{array}\right]=\left[\begin{array}{cc}h_{1} & h_{2} \\ h_{2}{ }^{*} & -h_{1}{ }^{*}\end{array}\right]\left[\begin{array}{l}p_{1} \\ p_{2}\end{array}\right]+\left[\begin{array}{c}n_{1} \\ n_{2}{ }^{*}\end{array}\right]$

$\mathrm{c} 1$ and $\mathrm{c} 2$ are the first and second columns of the channel matrix. $c_{1}{ }^{H}$ represents transpose conjugate of $\mathrm{c} 1$. When multiplying $c_{1}{ }^{H}$ with $\mathrm{c} 2$, if the result is zero then $\mathrm{c} 1$ is orthogonal to $\mathrm{c} 2$. This is called an orthogonal channel matrix and is represented as:

$c_{1}{ }^{H} c_{2}=\left[\begin{array}{ll}h_{1}^{*} & h_{2}\end{array}\right]\left[\begin{array}{c}h_{2} \\ -h_{1}^{*}\end{array}\right]=0$

To retrieve the transmitted symbols, weight vectors are used that are denoted as $W 1$ and $W 2$

$w_{1}=\frac{c_{1}}{\left\|c_{1}\right\|}=\frac{1}{\|h\|}\left[\begin{array}{c}h_{1} \\ h_{2}{ }^{*}\end{array}\right]$
$w_{2}=\frac{c_{2}}{\left\|c_{1}\right\|}=\frac{1}{\|h\|}\left[\begin{array}{c}h_{2} \\ -h_{1}{ }^{*}\end{array}\right]$

Equation (8) multiplied with the equation (5) to retrieve p1 symbol as:

$$
\begin{aligned}
& \begin{aligned}
w_{1}{ }^{H} \bar{y}= & \frac{1}{\|h\|}\left[\begin{array}{ll}
h_{1}{ }^{*} & h_{2}
\end{array}\right]\left[\begin{array}{cc}
h_{1} & h_{2} \\
h_{2}{ }^{*} & -h_{1}{ }^{*}
\end{array}\right]\left[\begin{array}{l}
p_{1} \\
p_{2}
\end{array}\right] \\
& +\overline{w_{1}}{ }^{H} \overline{n_{1}}
\end{aligned} \\
& =\frac{1}{\|h\|}\left[\left(h_{1}{ }^{*} h_{1}+h_{2} h_{2}{ }^{*}\right)\left(h_{1}{ }^{*} h_{2}-h_{1}{ }^{*} h_{2}\right)\right]\left[\begin{array}{l}
p_{1} \\
p_{2}
\end{array}\right] \\
& +\widetilde{n_{1}} \\
& =\frac{1}{\|h\|} p_{1}+\widetilde{n_{1}}
\end{aligned}
$$

Equation (9) multiplied with the equation (5) to retrieve the p2 symbol as:

$$
\begin{aligned}
& \begin{aligned}
w_{2}{ }^{H} \bar{y}= & \frac{1}{\|h\|}\left[h_{2}{ }^{*}-h_{1}\right]\left[\begin{array}{cc}
h_{1} & h_{2} \\
h_{2}{ }^{*} & -h_{1}{ }^{*}
\end{array}\right]\left[\begin{array}{l}
p_{1} \\
p_{2}
\end{array}\right] \\
& +\overline{w_{1}}{ }^{H} \overline{n_{1}}
\end{aligned} \\
& =\frac{1}{\|h\|}\left(h_{1}{ }^{*} h_{2}-h_{1}{ }^{*} h_{2}\right)\left(h_{1}{ }^{*} h_{1}+h_{2} h_{2}{ }^{*}\right)\left[\begin{array}{l}
p_{1} \\
p_{2}
\end{array}\right] \\
& +\widetilde{n_{2}} \\
& =\|h\| p_{2}+\widetilde{n_{2}}
\end{aligned}
$$

For a $2 \times 2 \mathrm{MIMO}$, the same process is used to retrieve the transmitted symbols using the OSTBC code, but complexity is increased due to an increase in one more antenna at the receiver [12], [13].

\section{IMPLEMENTATION OF 2 X 2 MIMO OFDM SYSTEM}

MIMO-OFDM system setup consists of 4-USRP's, 2 for transmitting and the other 2 for receiving signals. LabVIEW is a software in which block diagram is constructed with the inbuilt blocks of communication tools. To interface LabVIEW with USRP's Gigabit Ethernet router is used. 


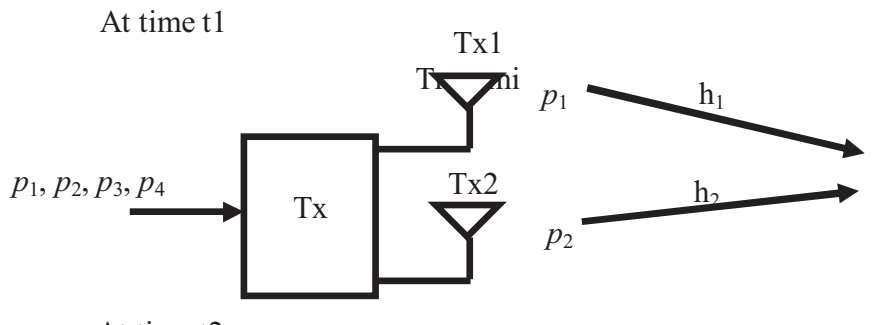

At time $\mathrm{t} 1$

At time $\mathrm{t} 2$
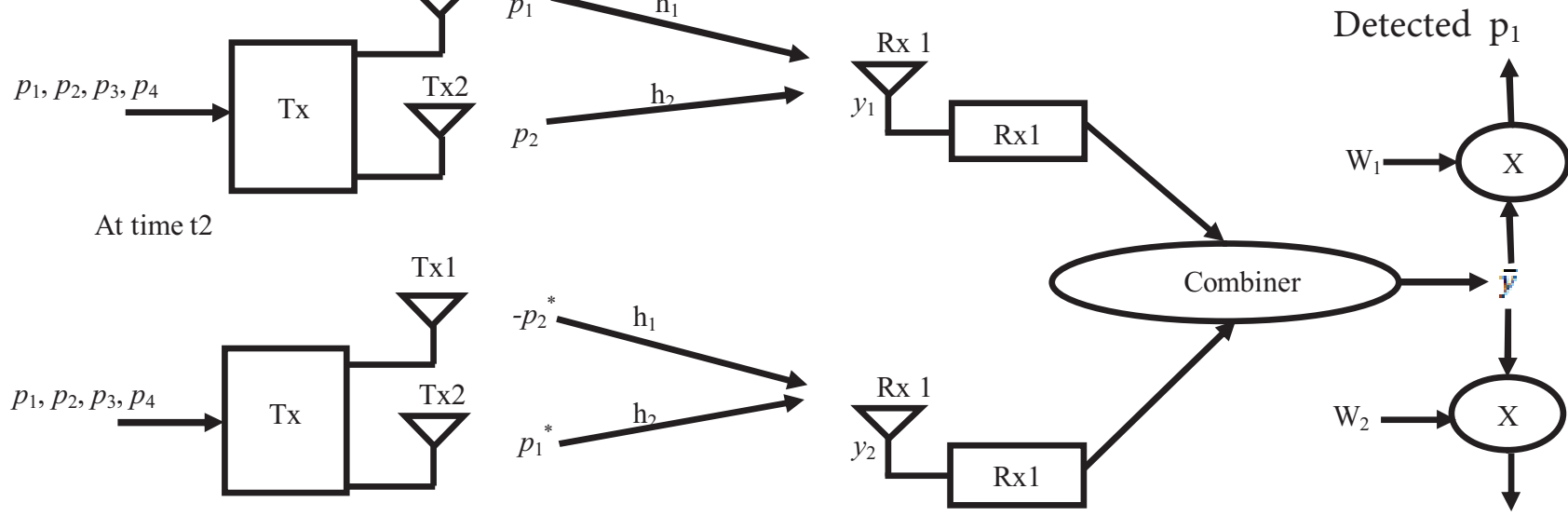

Figure 1. Alamouti code block diagram

Detected $\mathrm{p}_{2}$

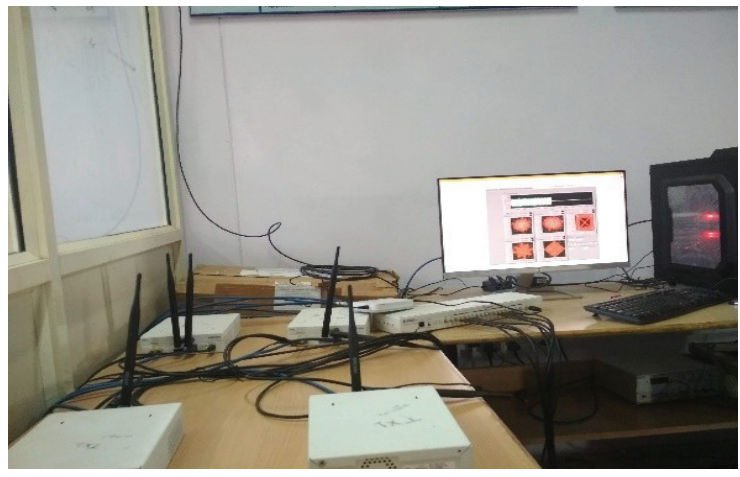

Figure 2. Experimental 2x2 MIMO setup

In MIMO system, the data is transmitted from two transmitters at the same time. For that, an octa-clock is used for synchronization between the transmitter and receiver. The physical connection between the devices is shown in Fig. 2. The signal is transmitted with a bandwidth of 30 $\mathrm{MHz}$ and a frequency of $2.4 \mathrm{GHz}$. In 2x2 MIMO-OFDM system implementation, the PN sequence is used to generate a random sequence as a message signal. The generated bits are modulated using M-QAM (4, 16, 64 QAM) and modulated symbols are feed to the MIMO encoding block that results in transmitted signals in two channels, for each channel OFDM technique is applied as shown in Fig. 3In OFDM, the wideband of serial data is converted into narrowband of $\mathrm{N}$ parallel data. Each narrowband is modulated with $\mathrm{N}$ subcarriers. Before the serial to parallel conversion of data, a training sequence (length 11 frank sequence) 0 is added for the channel estimation. The modulated subcarriers are passed through IFFT and the resultant OFDM signal is represented as follows:

$$
x_{n}=\frac{1}{\sqrt{N}} \sum_{k=0}^{N-1} X_{k} e^{\frac{j 2 \pi k n}{N}}, 0 \leq n \leq N
$$

The pulse shaping filter is used to reduce the bandwidth usage by clipping the edges and fit the signal into a transmitting channel.

The data is transmitted and received with the help of ADC in USRP. A maximum likelihood estimator is used for channel estimation. Schimidi cox algorithm is used for symbols timing recovery. The demodulation is used to recover the transmitted signal.

Fig. 4 shows the receiver of MIMO-OFDM in which a pulse shaping filter is used to receive the signal below the threshold level. The symbols in a signal get synchronized in symbol synchronization and the channel is estimated such that each channel constellation plot is obtained. To retrieve the Signal, the demodulated process is done.

\section{EXPERIMENTAL RESULTS AND DISCUSSIONS}

In LabVIEW front panel the results of $2 \times 1$ MIMO, $2 \times 2$ MIMO and 2x2 MIMO-OFDM are shown in figures 5-7. By using M-QAM modulation techniques, incoming bits are demodulated and an approximate constellation plot for two transmitting channels at the receiver is obtained. BER is calculated for the received signal and shown in Table II. The BER is estimated for $2 \times 1$ and 2x2 MIMO system with different M-ary QAM modulations and is given in Table II. From Table II, the $2 \times 2$ MIMO system BER is less. In the $2 \times 2$ MIMO system, 2 receivers receive the transmitted signals obtained from multiple paths and retrieve their respective transmitted signals. In a $2 x 1$ MIMO system, a single receiver can receive both the transmitted signals. The signals obtained at different delays cause interference, this results in high BER. 

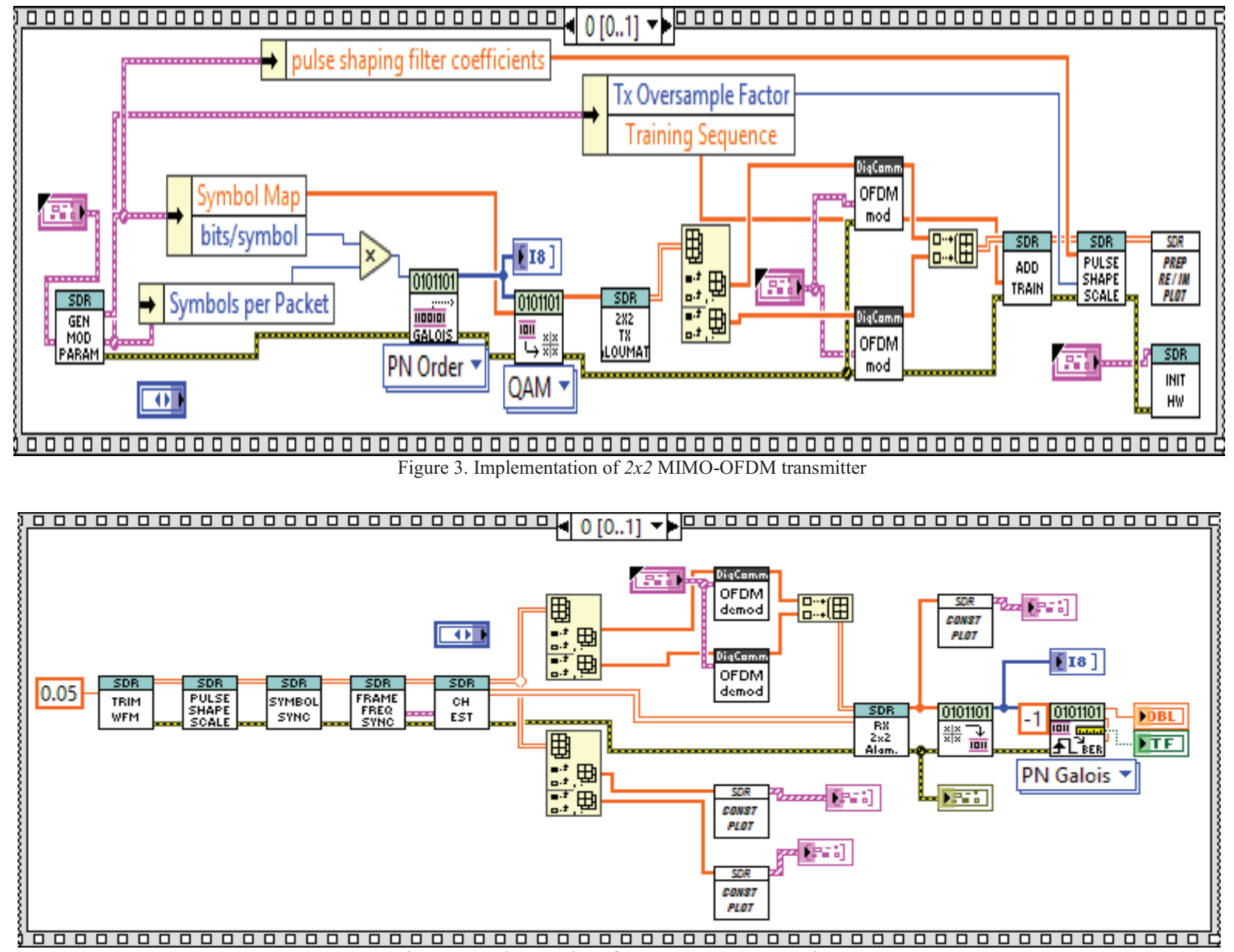

Figure 4. Implementation of $2 \times 2$ MIMO-OFDM receiver

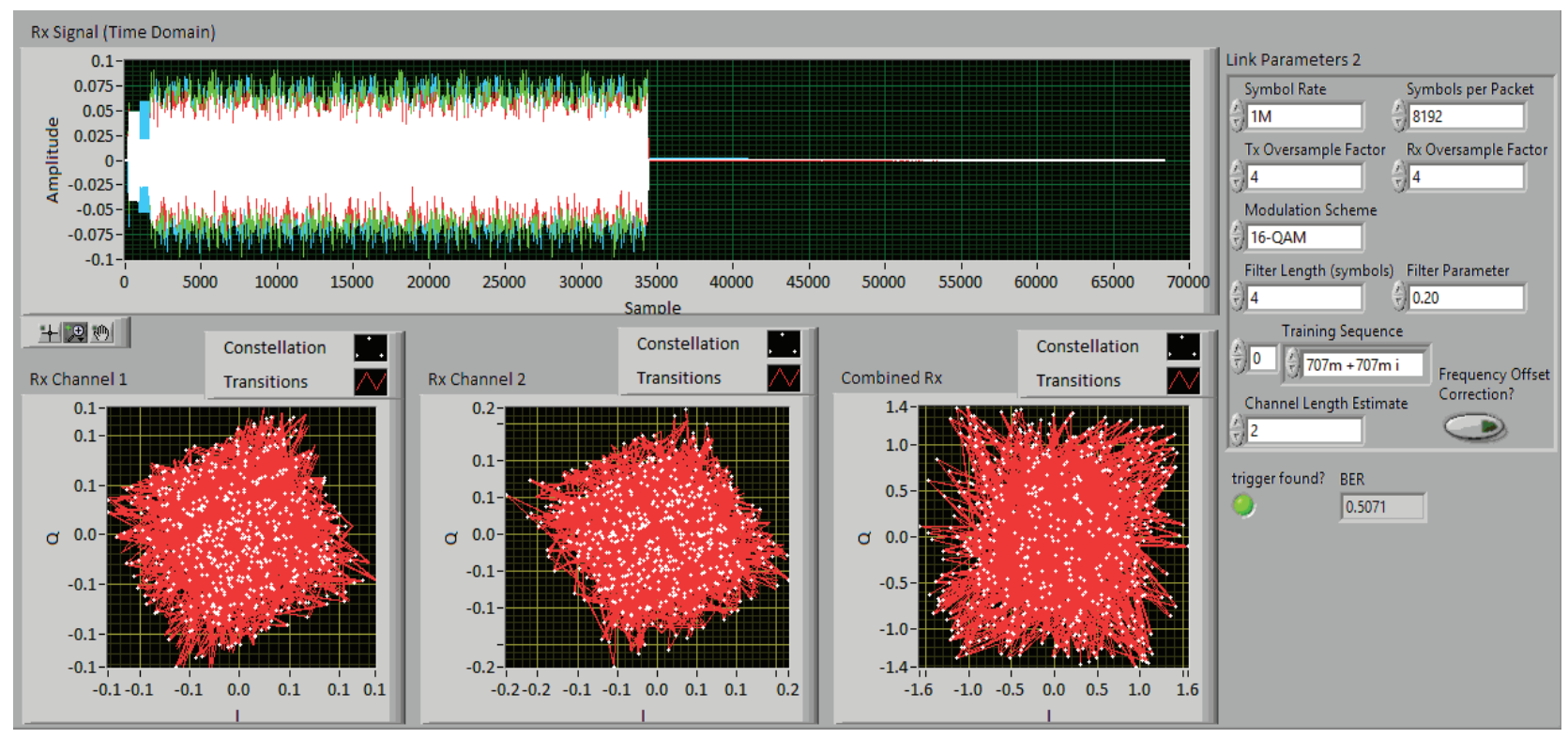

Figure 5. Front panel of 2X1 MIMO system 


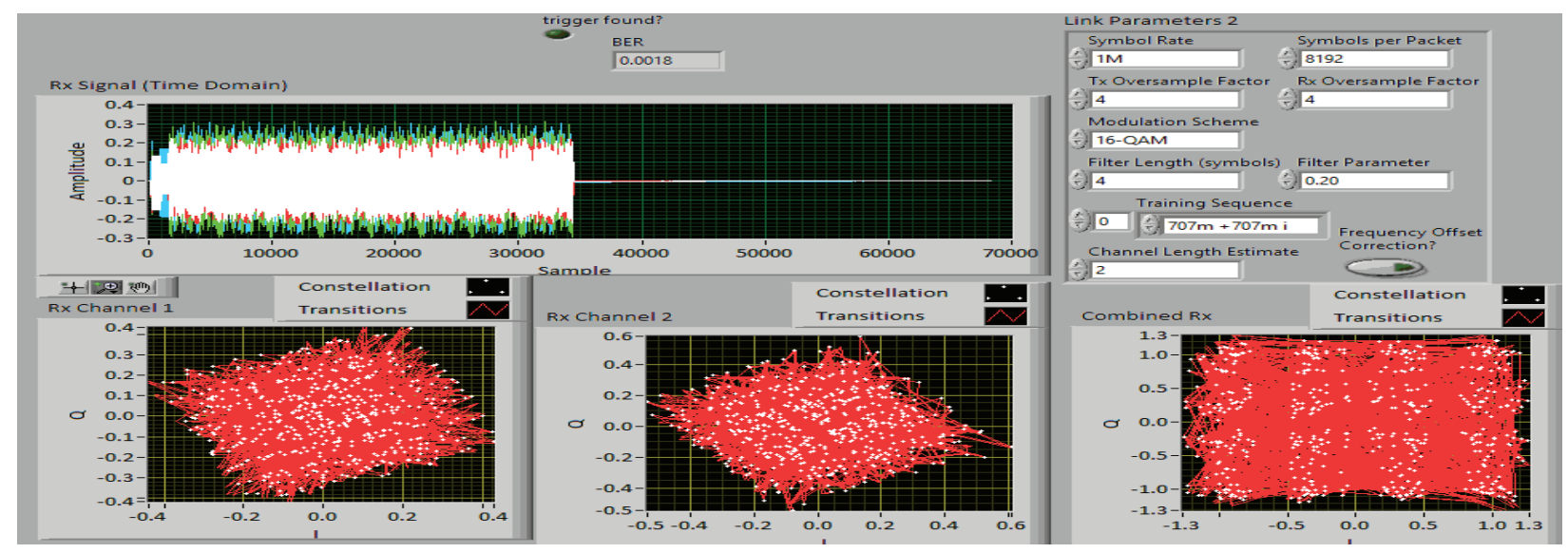

Figure 6. Front panel of $2 \times 2$ MIMO system

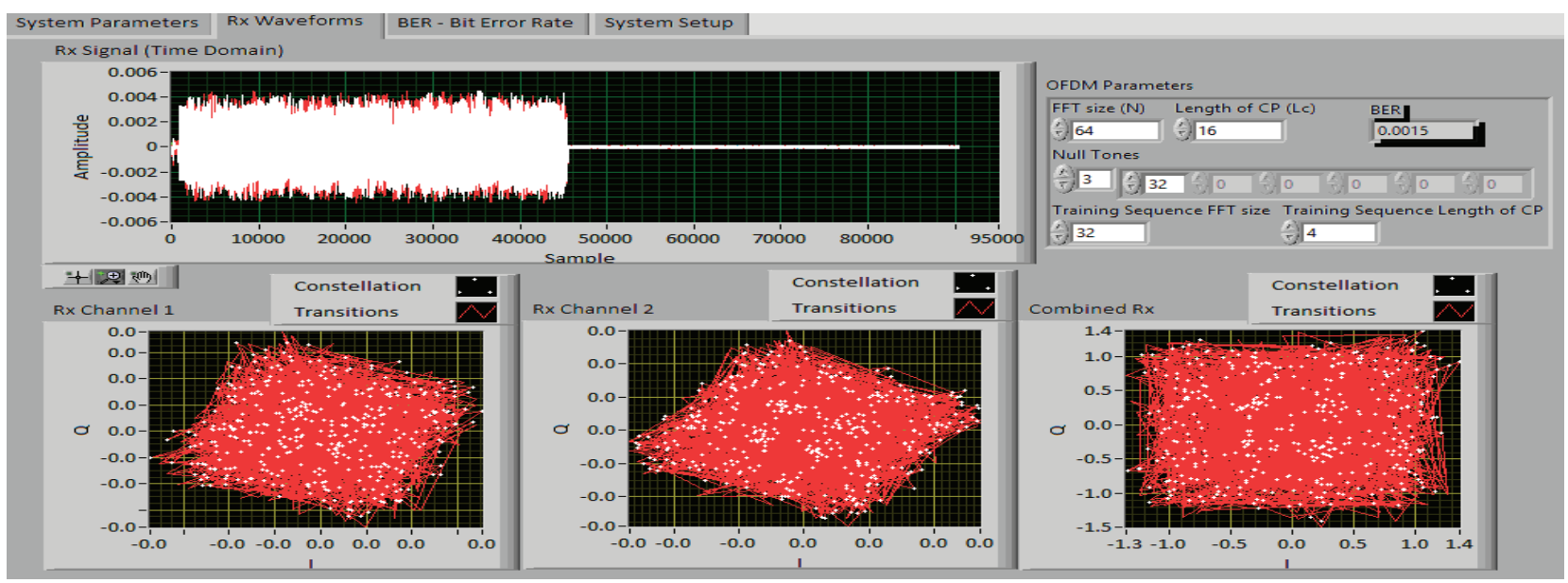

Figure 7. Front panel of $2 \times 2$ MIMO-OFDM system

TABLE II.

COMPARISON OF Ber PERFORMANCE of $2 x 1$ AND $2 x 2$

\begin{tabular}{|c|c|c|}
\hline \multirow{2}{*}{ QAM } & \multicolumn{2}{|c|}{ BER } \\
\cline { 2 - 3 } & $2 \times 1$ & $2 \times 2$ \\
\hline 4 & 0.0160 & 0.0000 \\
\hline 16 & 0.5071 & 0.0018 \\
\hline 64 & 0.9010 & 0.0701 \\
\hline
\end{tabular}

TABLE III.

COMPARISON OF BER PERFORMANCE AT DIFFERENT IFFT SIZES

\begin{tabular}{|c|c|r|c|}
\hline \multicolumn{4}{|c|}{$2 \times 2$ MIMO-OFDM } \\
\hline \multirow{2}{*}{ QAM } & \multicolumn{3}{|c|}{ BER with different IFFT } \\
\cline { 2 - 4 } & 32 & 64 & 128 \\
\hline 4 & 0.0002 & 0.0000 & 0.0008 \\
\hline 16 & 0.0039 & 0.0015 & 0.0028 \\
\hline 64 & 0.0593 & 0.0456 & 0.0818 \\
\hline
\end{tabular}

In order to increase spectral efficiency, the OFDM system is combined with the MIMO system to obtain better BER performance such that channel estimation of $2 \times 2$ MIMO-
OFDM system constellation is shown in Fig. 7 and resultant information about M-QAM with BER Performance is shown in Table III.

From Table III, BER for 64 IFFT is reduced, when compared to 128 IFFT because with increasing the number of IFFTs the Peak to Average Power Ratio (PAPR) gets increases. PAPR is calculated as the average peak of signal to maximum power. As increasing M-ary QAM modulation, the constellation points increase, and this causes interference between symbols and reduces the BER performance.

\section{Conclusions}

This paper includes the implementation of MIMO systems and their advantages. The main advantage of MIMO is high data rates and OFDM is high spectral efficiency. To capture this advantage, MIMO is combined with OFDM and MIMO-OFDM is implemented in LabVIEW using USRP. The BER of the MIMO-OFDM system is estimated as 0.0456 . With increase in the FFT size, BER also increases due to the increase in PAPR of the OFDM signal. $2 \times 2$ system is used in many applications includes IEEE 802.11n, IEEE 802.11a/g WLAN, Digital 
Video Broad Casting - Terrestrial $2^{\text {nd }}$ Generation (DVB-T2), $3^{\text {rd }}$ Generation Partnership Project (3GPP). It is also used in WiMAX which is the standard of IEEE 802.16, 5G and upcoming $6 \mathrm{G}$ technologies.

\section{REFERENCES}

[1] G Foschini, D Chizhik, M Gans, C Papadias and R Valen zuela,"Analysis and performance of some basic space-time architectures", IEEE Journal on Selected Area in Communications, Volume. 21, number. 3, pp.303-302, Apr. 2003.

[2] C F Ball, E Humburg, S Eder, L Lacinak, "WiMax Capacity Enhancements introducing MIMO 2x2 Diversity and Spatial Multiplexing", $200716^{\text {th }}$ IST Mobile and Wireless Communications Summit, Sep 2007.

[3] S. M. Alamouti, "A simple transmit diversity technique for wireless communications", IEEE Journal on Selected Areas Communications, Volume. 16, number. 8, pp. 1451-1458, Oct. 1998.

[4] Yong Soo Cho, Jaekwon Kim, Won Young Yang, and Chung G Kang, "MIMO-OFDM wireless communications with MATLAB", John Wiley \& Sons, 2010.

[5] Claude Oestges and Bruno Clerckx, "MIMO wireless communications: from real-world propagation to spacetime code design". Academic Press, 2010.

[6] A. Paulraj and T. Kailath, "Increasing capacity in wireless broadcast systems using distributed transmission/directional reception", Feb. 21, 1992, U.S. Patent 5345599.

[7] Yu Wei lin, Chen Yi Lee, "Design o an FFT/IFFT Processor for MIMO OFDM Systems", IEEE Transactions on Circuits and Systems, I: Regular Papers, Volume. 54, number 4, pp. 807-815, 16 April 2007.
[8] V Tarokh, H Jafarkhani, A R Calderbank, "Space-time block codes from orthogonal designs", IEEE Transactions on Information Theory, Volume. 45, number 5, pp. 1456-1467, July 1999.

[9] Pavan Kumar, Amita Kumari "BER Analysis of BPSK, QPSK, 16QAM \& 64-QAM based OFDM System over Rayleigh Fading Channel", IOSR Journal of Electronics and Communication Engineering, Volume. 11, number. 4, pp. 6674, Jul-Aug 2016.

[10] Malleswari Akurati, Yedukondalu Kamatham, Sathish Kumar Pentamsetty and Satya Prasad Kodati, "PAPR Reduction in OFDM using Hybrid Companding for 5G Wireless Communications", IEEE Global Conference for Advancement in Technology - GCAT 2019, Bengaluru, India, 18-20 October 2019.

[11] Malleswari Akurati, Yedukondalu Kamatham, Satish Kumar Pentamsetty and Satya Prasad Kodati, "Reduction of PAPR in OFDM USING Hybrid SLM-Companding for future Wireless Communications", IEEE Global Conference for Advancement in Technology- GCAT 2019, Bengaluru, India, 18-20 October 2019.

[12] Sravanti Thota, Yedukondalu Kamatham and Chandra Sekhar P, "Reduced Complexity Hybrid PAPR Reduction Schemes for Future Broadcasting Systems", International Conference on Emerging Trends in Engineering (ICETE), pp.69-76,22-23 March 2019.

[13] Sravanti Thota, Yedukondalu Kamatham and Chandra Sekhar P, "Performance Analysis of Hybrid Companding PAPR Reduction Method in OFDM Systems for 5G Communications", The $9^{\text {th }}$ International Conference on Computing, Communication and Networking Technologies (ICCCNT), pp. 1-5, July 10-12, 2018. 